\title{
Linkage disequilibrium and the genetic distance in livestock populations: the impact of inbreeding
}

\author{
Jérémie Nsengimana*, Philippe V. Baret** \\ Unité de génétique, Faculté d'ingénierie biologique, agronomique et environnementale, \\ Université catholique de Louvain, Croix du sud 2 box 14, 1348 Louvain-la-Neuve, Belgium
}

(Received 21 March 2003; accepted 22 January 2004)

\begin{abstract}
Genome-wide linkage disequilibrium (LD) is subject to intensive investigation in human and livestock populations since it can potentially reveal aspects of a population history, permit to date them and help in fine-gene mapping. The most commonly used measure of LD between multiallelic loci is the coefficient $D^{\prime}$. Data based on $D^{\prime}$ were recently published in humans, livestock and model animals. However, the properties of this coefficient are not well understood. Its sampling distribution and variance has received recent attention, but its expected behaviour with respect to genetic or physical distance remains unknown. Using stochastic simulations of populations having a finite size, we show that $D^{\prime}$ fits an exponential function having two parameters of simple biological interpretation: the residual value $(r s)$ towards which $D^{\prime}$ tends as the genetic distance increases and the distance $R$ at which this value is reached. Properties of this model are evaluated as a function of the inbreeding coefficient (F). It was found that $R$ and $r s$ increase when $\mathrm{F}$ increases. The proposed model offers opportunities to better understand the patterns and the origins of LD in different populations and along different chromosomes.
\end{abstract}

Linkage disequilibrium / livestock / inbreeding / genetic distance / exponential function

\section{INTRODUCTION}

Linkage (or gametic) disequilibrium is useful in revealing past genetically important events, in dating them and in fine-gene mapping $[1,2,4,8]$. However, the relationship between linkage disequilibrium (LD) and the genetic distance in different population structures is not well understood. For biallelic loci in a finite population and when LD is measured with $\Delta^{2}$, the squared correlation of allele frequencies (Eq. (1), where $p_{i}, q_{j}$ and $p_{i j}$ are respectively frequencies of

\footnotetext{
${ }^{*}$ Present address: Centre International de recherche sur le Cancer (CIRC), 150 cours Albert Thomas, 69008, Lyon, France

${ }^{* *}$ Corresponding author: baret@gena.ucl.ac.be
} 
alleles $i$ and $j$, and of the haplotype $i j$, see [10]), the expected value of LD at equilibrium drift-recombination is a function of the recombination rate $\theta$ and of the effective population size $N_{e}$ (Eq. (2), see [20]).

$$
\begin{aligned}
& \Delta^{2}=\frac{\left(p_{i j}-p_{i} q_{j}\right)^{2}}{p_{i} q_{j}\left(1-p_{i}\right)\left(1-q_{j}\right)} \\
& E\left[\Delta^{2}\right]=\frac{1}{1+4 N_{e} \theta} \cdot
\end{aligned}
$$

Although the original symbol used to represent the correlation of allelic frequencies is $\mathrm{r}^{2}$ [10], the symbol $\Delta^{2}$ is also commonly used (see e.g. [6]) and we chose this notation because $r^{2}$ will be used to measure the determination coefficient of a model fitting.

If the population size can be inferred and the equilibrium driftrecombination assumed, equation (2) expresses the LD as a function of the genetic distance. However, this model is expected to hold if LD results only from genetic drift (the initial linkage equilibrium is assumed) and if $4 N_{e} \theta>20$ [11]. In animal breeding, there may be a high initial level of LD resulting from the selection of a few breeding stocks that are crossed in half-sib designs by the way of artificial insemination or resulting from admixture. Due to this initial LD, equation (2) does not hold in most livestock populations. In addition, the validity of equation (2) is hampered by the fact that we do not have any information on whether there is equilibrium between drift and recombination in livestock populations.

Another limitation of equation (2) is that the interval of variation for the coefficient $\Delta^{2}$ depends on allelic frequencies. According to Lewontin [13], there is no measure of LD completely independent from allelic frequencies due to the nature of LD itself (i.e. the non-random allelic association). However, an appropriate standardisation can provide a measure of LD that has an interval of variation independent from allelic frequencies. Zapata and Visedo [23] demonstrated that, although the coefficient $\Delta^{2}$ is standardised, it varies from -1 to +1 if and only if allelic frequencies are similar at both loci, otherwise this interval is smaller. Evidence was given that, due to this fact, measuring LD with $\Delta^{2}$ can suggest a wrong relationship between LD and the genetic distance while making a true relationship undetectable [23]. Consequently, Zapata and Visedo [23] recommended to preferably use the coefficient $D^{\prime}$, whose interval of variation is allelic frequencies independent.

The coefficient $D_{i j}^{\prime}$ between two alleles $i$ and $j$ on two loci was defined by Lewontin [12] equations (4) to (6) where symbols have the same meaning as in 
equation (1) and extended to pairs of multiallelic loci by Hedrick [9] (Eq. (3) where $N_{A}$ and $N_{B}$ are numbers of alleles on loci $A$ and $B$ ).

$$
D^{\prime}=D_{A B}^{\prime}=\sum_{i}^{N_{A}} \sum_{j}^{N_{B}} p_{i} q_{j}\left|D_{i j}^{\prime}\right|
$$

with

$$
\begin{aligned}
D_{i j}^{\prime} & =\frac{D_{i j}}{D_{\max }} \\
D_{i j} & =p_{i j}-p_{i} q_{j}
\end{aligned}
$$

and

$$
\left\{\begin{array}{l}
D_{\max }=\min \left[p_{i} q_{j},\left(1-p_{i}\right)\left(1-q_{j}\right)\right] \text { if } D_{i j}<0 \\
D_{\max }=\min \left[\left(1-p_{i}\right) q_{j}, p_{i}\left(1-q_{j}\right)\right] \text { if } D_{i j}>0 .
\end{array}\right.
$$

Coefficients $D_{i j}^{\prime}$ and $D_{i j}$ (Eqs. (4) and (5)) can take positive and negative values, indicating that alleles are in a coupling or a repulsive state while $D_{A B}^{\prime}$ (Eq. (3)) takes only positive values. In the following sections, we use the notation $D^{\prime}$ for LD between pairs of multiallelic loci $\left(D_{A B}^{\prime}\right)$.

There is an increasing interest in the use of this coefficient in LD analyses at the chromosome or the whole genome level as well as in model animals such as Drosophila $[19,26]$, in livestock $[7,15,21]$ and in human populations $[18,25]$. The assessment of properties of this coefficient is requiring considerable attention (e.g. sampling distribution and variance, see [24]). However, the behaviour of $D^{\prime}$ against the genetic or physical distance has not been implicitly investigated and, as a consequence, estimates of $D^{\prime}$ between large sets of markers are difficult to interpret. It is not clear in which circumstance this coefficient is expected to correlate with the distance between markers. We describe hereunder a few empirical studies that dealt with this issue although no consensual conclusion has so far emerged.

McRae et al. [15] reported a significant negative correlation between $D^{\prime}$ and the genetic distance in domesticated sheep in New Zealand $(r=-0.34$, $P<0.001)$ whereas, using a similar marker density $(\sim 1$ per $10 \mathrm{cM})$, Tenesa et al. [21] did not find any such correlation in domesticated cattle in the United Kingdom. At a much finer scale (1 marker per 60 bp), Riley et al. [19] also failed to find a significant correlation between $D^{\prime}$ and the physical distance in Drosophila pseudoobscura $(r=-0.009, P>0.9)$.

Zapata et al. [25] found a weak but significant correlation between $D^{\prime}$ and the genetic distance on the human chromosome 11p15 ( $r=-0.226$, 
$P=0.037)$ while the correlation between $D^{\prime}$ and the physical distance was not significant $(r=-0.151, P=0.079$ ). With only pairs of coupling alleles (positive $\left.D_{i j}^{\prime}\right)$, this correlation was dependent on the allelic frequencies $(r=-0.192$, $P=0.019$ for alleles at frequency $>6 \%$ and $r=-0.284, P=0.017$ for alleles at frequency $>9 \%$ ). In the Holstein-Friesian dairy cattle, Farnir et al. [7] observed a decline of $D^{\prime}$ with the genetic distance but the significance of this correlation was not tested.

The objective of this study is to investigate the relationship between the coefficient of disequilibrium $D^{\prime}$ and the genetic distance and to assess the impact of inbreeding. The choice of $D^{\prime}$ is justified for several reasons: (1) it is a standardised measure of LD; (2) its interval of variation does not depend on allelic frequencies; (3) $D^{\prime}$ easily handles highly polymorphic loci such as microsatellites; and (4) data based on this parameter are increasingly available. The study makes extensive use of simulations. Hereafter, the material section describes the algorithm used to simulate various structures of populations and the methods section describes the approach used to estimate and fit LD as a function of the genetic distance. Then the obtained results are presented and discussed.

\section{MATERIALS AND METHODS}

\subsection{Material: simulated data}

We simulated four populations that mimic recently founded livestock populations (Tab. I). One male individual (the founder) was used to inseminate a large number of females (generation 1) and two hundred of these crosses gave one offspring each constituting then a second generation of 200 half sibs, with a sex ration of $1: 1$. In subsequent generations, a limited number of random crosses are simulated with a constant population size of 200 individuals per generation (Tab. I) and a sex ratio of 1:1.

In generation 1 of each population, fifty microsatellite markers were considered with six alleles each. They were evenly spaced on a $49 \mathrm{cM}$ chromosome. On each marker, the founder allele was drawn randomly from the set of six with a uniform distribution. The founder haplotype given to each offspring was drawn randomly from a Bernouilli distribution with a frequency of 0.5 and a recombination rate assuming the absence of interference (Haldane mapping function). Since an infinite number of dams was assumed and each dam had one offspring, the haplotypes of the dams were not constructed. The maternal allele given to the offspring at each marker was drawn randomly from a set of six with a uniform distribution. The simulated designs corresponded then to 
Table I. Structures of simulated populations.

\begin{tabular}{lccccc}
\hline \multirow{2}{*}{ Population } & \multicolumn{2}{c}{ Generation 1 to 2 } & \multicolumn{2}{c}{ Generation 2 to 10 } & \multirow{2}{*}{ Population size } \\
& Founder & Offspring & Crosses & Offspring & \\
\hline Pop4 & 1 & 200 & 4 & 50 & 200 \\
Pop10 & 1 & 200 & 10 & 20 & 200 \\
Pop25 & 1 & 200 & 25 & 8 & 200 \\
Pop100 & 1 & 200 & 100 & 2 & 200 \\
\hline
\end{tabular}

linkage equilibrium in the founding generation and strong linkage disequilibrium in the following generation of half sibs.

Starting at the generation of half sibs (generation 2), paternal and maternal haplotypes transmitted to offspring were drawn randomly from a Bernouilli distribution with a frequency of 0.5 and a recombination rate based on the Haldane mapping function (assuming the absence of interference). For each population, 10 generations were simulated with 1000 replicates.

\subsection{Methods}

The inbreeding coefficient and kinship coefficients were computed iteratively using the records of pedigree information, according to Lynch and Walsh [14]. The mean inbreeding coefficient $(F)$ was computed at each generation for each population. From the rate of inbreeding $(\Delta F)$ between generation 9 and 10, we estimated the population effective sizes $\left(N_{e}\right)$ with the relationship

$$
\Delta F=\frac{1}{2 N_{e}+1}
$$

At generation 10, equations (3) to (6) were used to estimate $D^{\prime}$ between all possible pairs of markers (1225 pairs) with 400 haplotypes, for each of the 1000 simulations within each of the four populations. It was assumed that the linkage phase of different alleles is known in the analysed generation. In practice, linkage phases are constructed from genotypes of progeny, their parents and their grandparents if available (see e.g. $[7,15]$ ).

For each of the 1000 simulations, estimates of $D^{\prime}$ were plotted against the genetic distance and a least squares approach was applied to fit an exponential function (Eq. (8)) to this spatial pattern

$$
D^{\prime}(x)=r s+(1-r s) \exp \left(\frac{-3 x}{R}\right)
$$


This spatial model stipulates that the highest value of $D^{\prime}$ is 1 and it corresponds to the genetic distance $(x)$ zero. As the distance increases, $D^{\prime}$ decreases until a residual value $(r s)$ is reached. The parameter $R$ should correspond to the distance at which $D^{\prime}$ drops to $r s$. However, since equation (8) is an asymptotic function, we follow a convention of spatial data modelling (see e.g. [5]): we estimate $R$ as the distance at which the spatially correlated part of $D^{\prime}$ drops to $5 \%$ [i.e. $D^{\prime}=r s+0.05(1-r s)$ ]. In fact, the exponential function is one of the models used in spatial data analysis [5] and we used it to fit LD owing to the known exponential relationship between the genetic recombination and the genetic distance (the Haldane mapping function was used in data simulation).

\section{RESULTS}

\subsection{Inbreeding coefficient}

In the base population, all individuals were assumed to be unrelated. In the second generation, offspring of the founder were half sibs and the inbreeding coefficient between any two of them was equal to zero. In generation 3, all individuals had at least one common grandparent (the founder) so that the mean inbreeding coefficient $(\mathrm{F})$ is equal to 0.125 in all four populations. From generation 4 to 10, the rate of increase in F depends on the mating structure (Fig. 1). The effective population sizes at generation 10 were 13.0, 24.5, 49.5 and 166.2 in pop4, pop10, pop25 and pop100, respectively.

\subsection{Allele frequencies in generation 10}

Amongst the six alleles simulated per marker in the base generation, on average 2.61 to 5.90 remain 10 generations later according to the population (Tab. II). As expected, the proportion of these mean alleles per marker decreases with the increase of the inbreeding coefficient $\left(\mathrm{F}_{10}\right)$.

The frequency distribution of these alleles is also a function of inbreeding: while there are no alleles with a frequency greater than 0.80 in pop100 $\left(\mathrm{F}_{10}=\right.$ 0.15 ), they appear progressively at the expense of low and medium frequencies as the inbreeding increases (Fig. 2). The allelic fixation is observed in the most inbred populations (up to $3 \%$ in pop4, $\mathrm{F}_{10}=0.43$ ).

\subsection{Estimates of $D^{\prime}$ and their spatial pattern}

Ten generations after populations were founded, the distribution of $D^{\prime}$ between all pairs of markers depends on the inbreeding. In the less inbred 


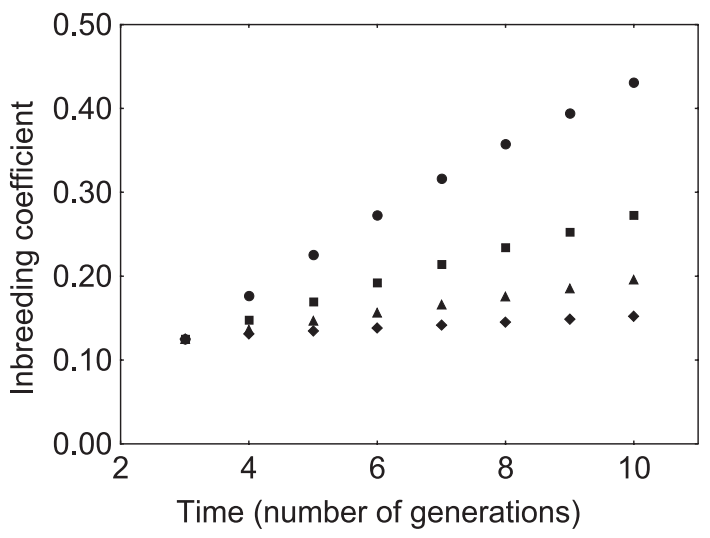

Figure 1. Mean inbreeding coefficient across generations in the four simulated populations. From top to bottom: pop4, pop10, pop25 and pop100.

Table II. Mean number of alleles per marker $\left(N_{A}\right)$ in generations 10 .

\begin{tabular}{lcc}
\hline Population & $\mathrm{F}_{10}$ & $N_{A} \pm \sigma$ \\
\hline Pop100 & 0.15 & $5.90 \pm 0.03$ \\
Pop25 & 0.20 & $5.42 \pm 0.19$ \\
Pop10 & 0.27 & $4.01 \pm 0.41$ \\
Pop4 & 0.43 & $2.61 \pm 0.41$ \\
\hline
\end{tabular}

populations (pop100 with $\mathrm{F}_{10}=0.15$ and pop25 with $\mathrm{F}_{10}=0.20$ ), the distribution is unimodal and asymmetric with the highest frequency of $D^{\prime}$ in the interval $0.20-0.30$ (Fig. 3). As inbreeding increases, this distribution is flattened and extreme values of $D^{\prime}$ appear progressively (down to 0 and up 1). These tail values represent $\sim 30 \%$ of the distribution in pop4 $\left(\mathrm{F}_{10}=0.43\right)$.

Equation (8) was applied to $D^{\prime}$ in each of the 1000 simulations of every population. The adequacy of the model (indicated by the determination coefficient, $r^{2}$ ) depends on the inbreeding: $r^{2}$ decreases when the inbreeding coefficient increases (Tab. III). In the less inbred populations (pop100 and pop25 with $\mathrm{F}_{10}=0.15$ and 0.20 respectively), $\mathrm{r}^{2}$ is greater than 0.50 in all simulations (Tab. III). On the contrary, $\mathrm{r}^{2}$ is lower than 0.50 in $33 \%$ of simulations of pop10 $\left(\mathrm{F}_{10}=0.27\right)$ and in $95 \%$ of the simulations of pop4 $\left(\mathrm{F}_{10}=0.43\right)$.

A poor fitting of the spatial model to $D^{\prime}$ in highly inbred conditions is caused by extreme values of $D^{\prime}=1$ and $D^{\prime}=0$ observed between loci separated by various genetic distances. Figure 4 illustrates two examples of simulations from pop4 with a poor fitting. The parameters were respectively $R=31 \mathrm{cM}$, 


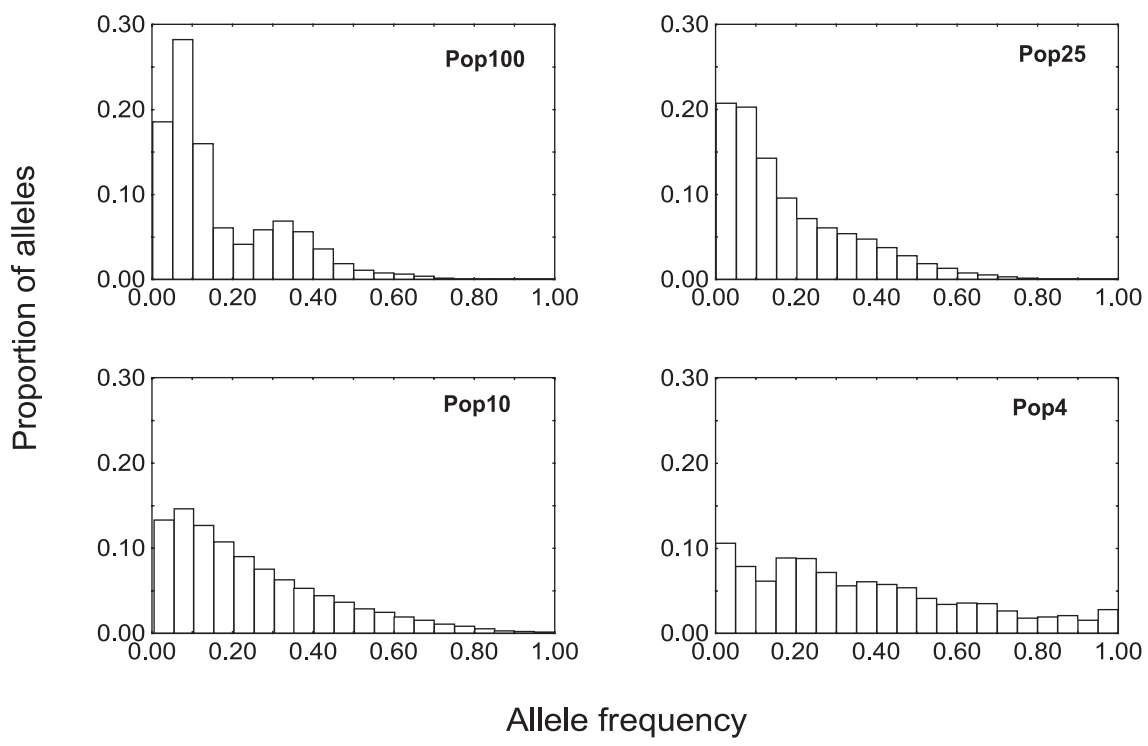

Figure 2. Frequency distribution of remaining alleles at generation 10 for 1000 simulations. The inbreeding coefficient $\left(\mathrm{F}_{10}\right)$ is respectively 0.15 in pop100, 0.20 in pop 25 , 0.27 in pop10 and 0.43 in pop4.
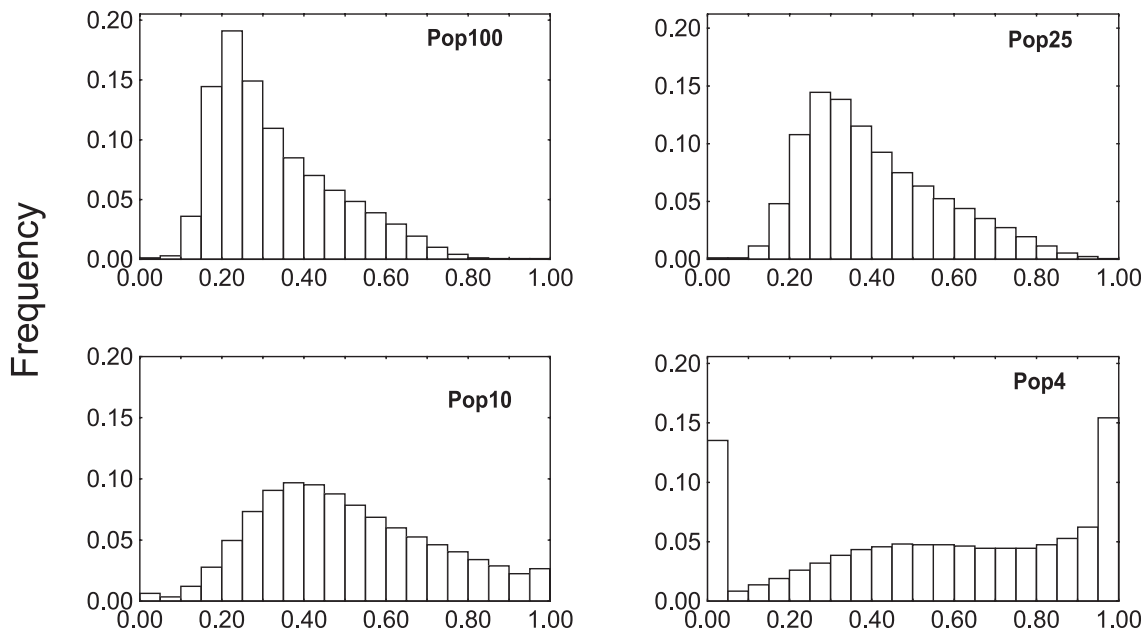

Estimate of $D^{\prime}$

Figure 3. Distribution of $D^{\prime}$ in 1000 simulations at generation $10 . \mathrm{F}_{10}$ is respectively 0.15 in pop100, 0.20 in pop25, 0.27 in pop10 and 0.43 in pop4. 
A

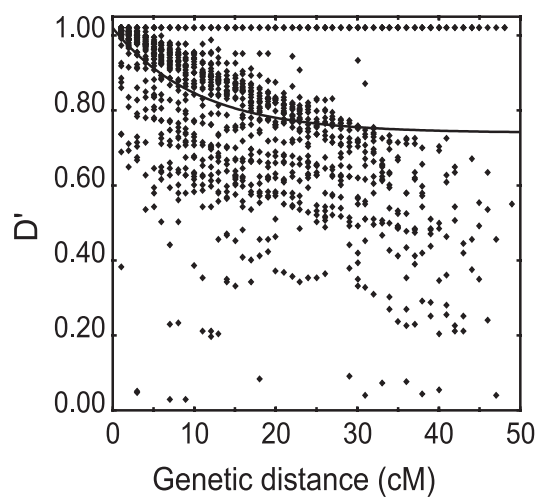

B

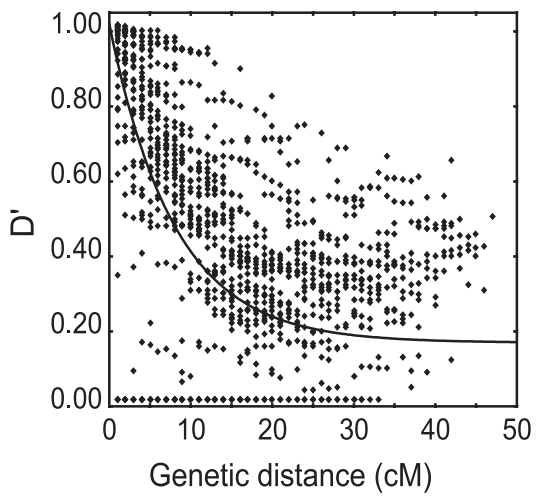

Figure 4. Example of simulations of pop4 with a poor fitting of the model. For A, 159 pairs of markers over $1225(13 \%)$ have $D^{\prime}=1$ and parameters of the exponential function are $R=31 \mathrm{cM}$ and $r s=0.72$ with $\mathrm{r}^{2}=0.10$. In B, 97 pairs on $1225(8 \%)$ have $D^{\prime}=0$ and parameters of the exponential model are $R=24 \mathrm{cM}$ and $r s=0.15$ with $\mathrm{r}^{2}=0.19$.

Table III. Mean determination coefficient $\left(\mathrm{r}^{2}\right)$ in generation 10 .

\begin{tabular}{lccr}
\hline Population & $\mathrm{F}_{10}$ & $\mathrm{r}^{2} \pm \sigma$ & $\mathrm{r}^{2}<0.50^{\S}$ \\
\hline Pop100 & 0.15 & $0.77 \pm 0.04$ & $0 \%$ \\
Pop25 & 0.20 & $0.69 \pm 0.06$ & $0 \%$ \\
Pop10 & 0.27 & $0.53 \pm 0.11$ & $33 \%$ \\
Pop4 & 0.43 & $0.24 \pm 0.14$ & $95 \%$ \\
\hline
\end{tabular}

$\S$ Proportion of simulations with $\mathrm{r}^{2}$ lower than 0.50 .

$r s=0.72$ with $\mathrm{r}^{2}=0.10$ in Figure $4 \mathrm{~A}$ and $R=24 \mathrm{cM}, r s=0.15$ with $\mathrm{r}^{2}=0.19$ in Figure 5B.

In simulations without extreme values of $D^{\prime}(0$ or 1$)$ at large genetic distance, the model adequately fitted data in all four populations. Figure 5 illustrates two examples of simulations where the model fitted data with $\mathrm{r}^{2}>0.50$. The simulation of Figure $5 \mathrm{~A}$ is from pop4 and the corresponding $\mathrm{r}^{2}$ is 0.58 while Figure 5B is from pop100 and the corresponding $r^{2}$ is 0.84 .

In many simulations, the poor fitting was caused by a small proportion of observations: in Figure 4A and 4B there are only 13\% and 8\% extreme values of $D^{\prime}=1$ and 0 , respectively. Therefore, the model may not be considered as inappropriate. It may be preferable to exclude these tail values and fit the overall pattern of remaining observations. In this prospective, we observed a 

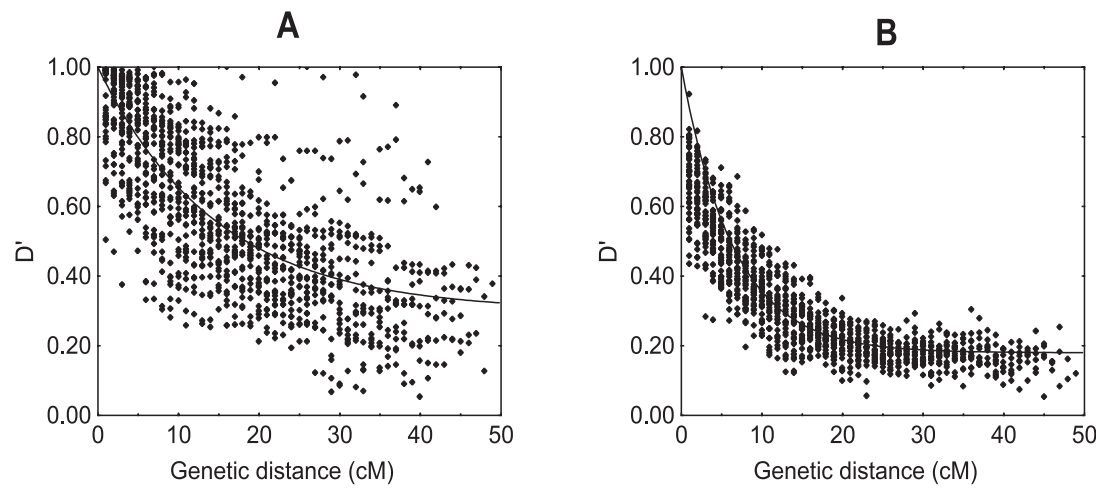

Figure 5. Example of simulations of pop4 (A) and pop100 (B) with adequate fitting. In $\mathrm{A}$, the parameters of the model used are $R=44 \mathrm{cM}$ and $r s=0.30$ with $\mathrm{r}^{2}=0.58$; and in $\mathrm{B}$, these parameters are $R=19.5 \mathrm{cM}, r s=0.18$ and $\mathrm{r}^{2}=0.84$.

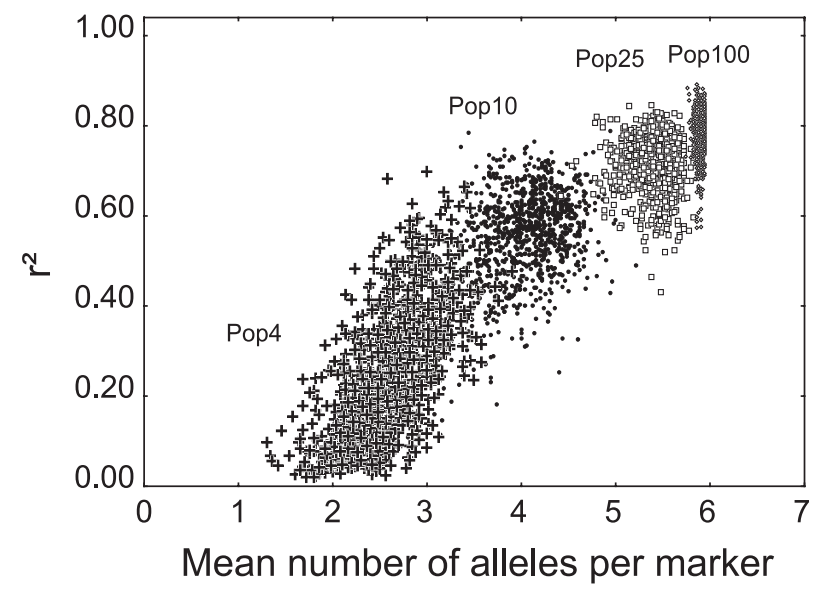

Figure 6. Determination coefficient $\left(\mathrm{r}^{2}\right)$ as a function of the mean number of alleles per locus in generation 10 .

positive correlation between $r^{2}$ and the mean number of alleles per marker within and between populations (Fig. 6). Since the reduction of the number of alleles is caused by the high level of inbreeding, we expected this relationship.

Therefore, to reduce the proportion and the impact of extreme values of $D^{\prime}$, we considered two criteria based on the number of alleles segregating per marker. As the first criterium, we retained simulations in which there are at least 3 alleles segregating on a minimum number of 5 markers covering a minimum length of $25 \mathrm{cM}$. The second criterium was more stringent in that 
Table IV. Parameters of the exponential function applied to $D^{\prime}$ with criteria based on marker polymorphism (generation 10).

\begin{tabular}{|c|c|c|c|c|c|}
\hline & Population & Pop100 & Pop25 & Pop10 & Pop4 \\
\hline & $\mathrm{F}_{10}$ & 0.15 & 0.20 & 0.27 & 0.43 \\
\hline \multirow{6}{*}{$\begin{array}{l}\text { Criterium } \\
1(\geq 3 \\
\text { alleles per } \\
\text { marker) }\end{array}$} & $R \pm \sigma(\mathrm{cM})$ & $17.6 \pm 2.3$ & $21.3 \pm 3.7$ & $30.5 \pm 8.4$ & $45.8 \pm 18.2$ \\
\hline & $r s \pm \sigma$ & $0.22 \pm 0.02$ & $0.28 \pm 0.03$ & $0.34 \pm 0.06$ & $0.37 \pm 0.11$ \\
\hline & $\mathrm{r}^{2} \pm \sigma$ & $0.78 \pm 0.04$ & $0.70 \pm 0.05$ & $0.59 \pm 0.07$ & $0.62 \pm 0.12$ \\
\hline & $\mathrm{r}^{2}<0.50^{\S}$ & $0 \%$ & $0 \%$ & $11 \%$ & $16 \%$ \\
\hline & $\begin{array}{l}\text { Number of } \\
\text { simulations }\end{array}$ & 1000 & 1000 & 936 & 567 \\
\hline & $\begin{array}{l}\text { Mean number of } \\
\text { markers }\end{array}$ & 49.6 & 49.6 & 47.8 & 30.8 \\
\hline \multirow{6}{*}{$\begin{array}{l}\text { Criterium } \\
2(\geq 4 \\
\text { alleles per } \\
\text { marker })\end{array}$} & $R \pm \sigma(\mathrm{cM})$ & $17.1 \pm 2.3$ & $21.3 \pm 3.7$ & $32.7 \pm 10.9$ & $57.7 \pm 28.6$ \\
\hline & $r s \pm \sigma$ & $0.22 \pm 0.02$ & $0.28 \pm 0.03$ & $0.36 \pm 0.08$ & $0.43 \pm 0.16$ \\
\hline & $r^{2} \pm \sigma$ & $0.78 \pm 0.04$ & $0.72 \pm 0.05$ & $0.73 \pm 0.10$ & $0.73 \pm 0.12$ \\
\hline & $\mathrm{r}^{2}<0.50^{\S}$ & $0 \%$ & $0 \%$ & $0 \%$ & $0 \%$ \\
\hline & $\begin{array}{l}\text { Number of } \\
\text { simulations }\end{array}$ & 1000 & 1000 & 836 & 10 \\
\hline & $\begin{array}{l}\text { Mean number of } \\
\text { markers }\end{array}$ & 48.8 & 45.4 & 18.3 & 5.2 \\
\hline
\end{tabular}

$\S$ Proportion of simulations with $\mathrm{r}^{2}$ lower than 0.50 .

a minimum number of 4 alleles per marker are required, all other conditions remaining unchanged ( $\geq 5$ markers covering $\geq 25 \mathrm{cM}$ ).

With the first criterium, the proportion of simulations having $\mathrm{r}^{2}<0.50$ decreased from $95 \%$ to $16 \%$ in pop4 and from $33 \%$ to $11 \%$ in pop10 (Tabs. III and IV) while the mean $\mathrm{r}^{2}$ was higher than 0.59 in all four populations (Tab. IV). However, the number of markers per chromosome fulfilling this criterium decreased with the increase of inbreeding. In the two more inbred populations (pop4 and pop25), the number of simulations also decreased but remained higher than 560. When the second criteria was applied, the model fitted data with $\mathrm{r}^{2}>0.50$ in all simulations, with an average always higher than 0.70 (Tab. IV).

In the two less inbred populations (pop25 and pop100), the results obtained $\left(R, r s\right.$, their standard deviation and $\left.r^{2}\right)$ were similar under both criteria (Tab. IV). This was not surprising since the two criteria can be taken as equivalent in these two populations given the average number of alleles $>5$ per marker (Tab. II). In pop10, the average number of alleles was 4 per marker 

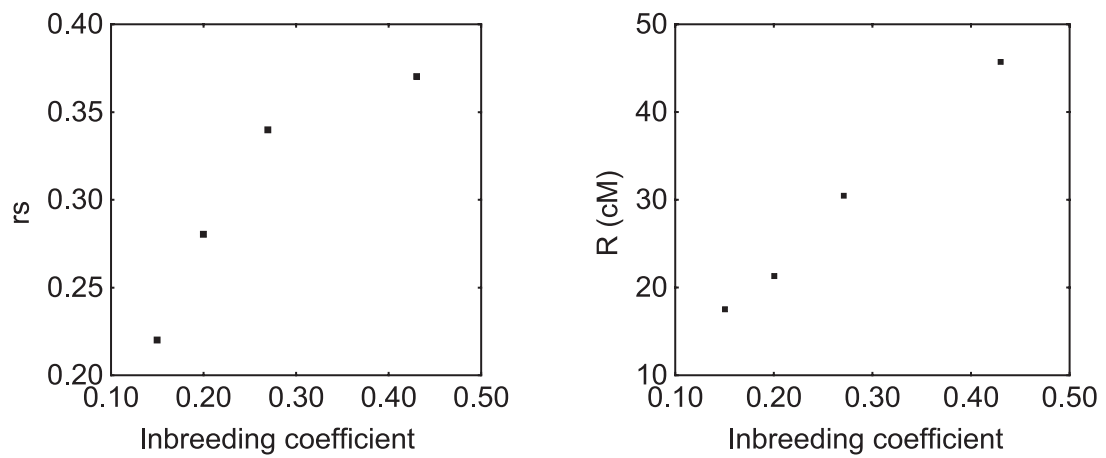

Figure 7. Parameters $R$ and $r s$ obtained with criterion 1 (more than 2 alleles per marker) as a function of the inbreeding coefficient at generation 10.

(see Tab. II) and the second criterium dramatically reduced the number of markers (only 18/50 were remaining), yet the estimates of parameters $R$ and $r s$ were similar to those obtained with criterium 1 (see Tab. IV). In pop4, there was a discrepancy between the results of the two criteria $(R, r s$, their standard deviations and $\mathrm{r}^{2}$ ), which can be attributed to the low numbers of simulations and markers per chromosome fulfilling the second criterium (10 simulations with 5.2 markers per chromosome).

\subsection{Effect of inbreeding on $R$ and $r s$}

The results of our analyses suggest a positive correlation between $R$ and $r s$ on the one hand and the inbreeding coefficient on the other hand (Tab. IV). With data obtained under the first criterion ( $\geq 3$ alleles/marker), the correlation between $R$ and $\mathrm{F}$ is high and significant ( $\mathrm{r}=0.98$ and $P<0.01$, Fig. 7). The correlation between $r s$ and $\mathrm{F}$ is also high but it is not significant $(\mathrm{r}=0.91$, $P=0.08$ ).

The variance of parameters $R$ and $r s$ also depends on the inbreeding: with the first criterion, the coefficient of variation (CV) is the lowest in pop100 (13\% for $R$ and $9 \%$ for $r s, \mathrm{~F}_{10}=0.15$ ) and it is the highest in pop4 (40\% for $R$ and $30 \%$ for $r s, \mathrm{~F}_{10}=0.43$ ).

\section{DISCUSSION}

Linkage disequilibrium is a useful characteristic of populations. However, its properties are not fully known in diverse population structures. Though it 
is feasible to estimate LD at the whole genome scale, it has not yet been possible to systematically characterise populations according to this parameter. A major issue around the quantification of LD is the choice of which measure to use from the large panoply of existing coefficients and approaches (see reviews of [6] and [9]). Since $D^{\prime}$ is the most common measure of LD between multiallelic markers, it is worth investigating its properties. In this study, we used an exponential function of the genetic distance (Eq. (8)) to fit $D^{\prime}$ and we assessed the properties of this function in inbred populations. These properties are parameters of the model $(R$ and $r s)$ and the determination coefficient $\left(\mathrm{r}^{2}\right)$ as an indicator of the goodness-of-fit.

The two parameters of the exponential function that we used (Eq. (8)) have a simple biological interpretation: $r s$ is the residual LD and $R$ is the distance at which $D^{\prime}$ drops to $r s$. These parameters may give an indication on the population demographic history: when LD is maintained in populations by a genetic drift, both $R$ and $r s$ are correlated to the inbreeding coefficient (Fig. 7). As a high inbreeding coefficient $(\mathrm{F})$ is caused by a small population effective size $\left(N_{e}\right)$, the positive correlation between $R$ and $r s$ on the one hand and $\mathrm{F}$ on the other hand means that $R$ and $r s$ are negatively correlated to $N_{e}$. We choose to characterise linkage disequilibrium with respect to $\mathrm{F}$ because it is close to other parameters such as identity by descent that are used in QTL mapping. However, the accuracy of estimates of $R$ and $r s$ also depends on inbreeding: with an inbreeding coefficient varying from 0.15 to 0.43 , the coefficient of variation (CV) varies between $13 \%$ and $40 \%$ for $R$ and between $9 \%$ and $30 \%$ for $r s$ when criterion 1 was used, i.e. at least 3 alleles segregating per marker.

In the four simulated populations, we considered a high level of LD at the time they were founded and the model used to fit LD (Eq. (8)) does not assume a final equilibrium between drift and recombination, on the contrary to the model of Hill and Robertson ([10], see Eq. (2)). Pop25 and pop100 may correspond to realistic livestock populations, since effective sizes were 49.5 and 166.2. Boichard et al. [3], Moureaux et al. [16] and Nagamine et al. [17] reported values of the same order in domesticated cattle and pigs. Pop4 and pop10 were simulated in order to assess the behaviour of the model used in particular conditions of very small populations $\left(N_{e}\right.$ was respectively 13.0 and 24.5).

In ideal populations, LD should only be influenced by genetic recombination. Given the exponential relationship between genetic recombination and genetic distance (the Haldane or Kosambi mapping function), it is expected that LD be related to recombination (or genetic distance) through an exponential relationship. In inbred populations, genetic recombinations can happen 
between chromosome segments that are homozygous; giving therefore recombined haplotypes that are identical to the non-recombined ones. As a result, inbreeding makes the relationship between LD and genetic distance more difficult to predict. The model that we used is appropriate in realistic settings since $\mathrm{r}^{2}$ was greater than $50 \%$ in all 1000 simulations of pop25 and pop100 when all markers were analysed (Tab. III). In more inbred conditions, the model was sensitive to extreme values of $D^{\prime}$ (0 or 1$)$ even when they represented a small proportion of observations (see Fig. 4). In such conditions, the real pattern of $D^{\prime}$ was adequately fitted when such values were excluded.

Between two alleles, extreme values of $D_{i j}^{\prime}$ can be caused by very small allelic frequencies and it is common to exclude these rare alleles from analyses in order to reduce their impact (i.e. arbitrary fixation of a lower bound of allelic frequencies to analyse, see e.g. $[18,25])$. However, in the multiallelic $D^{\prime}$, the impact of rare alleles is limited by using weights in the summation (Eq. (3)). This explains why we did not observe high values of $D^{\prime}$ in pop100 (where there are many rare alleles) and we observed them in pop4 (where there are fewer rare alleles). The distributions of allelic frequencies and of $D^{\prime}$ (Figs. 2 and 3 ) suggest that extreme values of $D^{\prime}$ in our populations were obtained between pairs of loci; one of which (at least) had an extremely frequent allele (fixed or nearby fixation). Since this also implies a reduction of the number of segregating alleles per marker (see Tab. II and Fig. 6), we found that an appropriate strategy to reduce the impact of extreme values of $D^{\prime}$ is to sufficiently analyse polymorphic markers. In fact, our study was primarily concerned with multiallelic loci for which LD is the most difficult to interpret.

The approach used in this study can be applied to large sets of microsatellite data that are available, from the experiments of QTL mapping. In fact, most LD studies exploit data that were generated in a purpose of linkage analysis, e.g. [7, 15,21]. Taking profit of these data and using parameters $R$ and $r s$ can provide more insight in our understanding of sources of LD by comparing for example populations or chromosomes. Since there is an increasing interest in the use of single nucleotide polymorphisms (SNP) [22], it will be of interest to evaluate properties of equation (8) with these biallelic data in various population structures. We performed this analysis for the case of pop100 and we obtained the same result as with microsatellite data: for 1000 simulations, the model fitted $D^{\prime}$ between SNPs at generation 10 with average parameters of $R=16.2+2.7 \mathrm{cM}, r s=0.24+0.03$ and $\mathrm{r}^{2}=0.72+0.04$. This result is an indication that in pop4 and pop10, a low accuracy in estimating $R$ and $r s$ was caused by inbreeding rather than by marker polymorphism. 


\section{ACKNOWLEDGEMENTS}

We are grateful for the support of the Belgian Fund for Research in Agriculture and Industry (FRIA), the National Fund for Scientific Research (FNRS) and the FSR program of the Université Catholique de Louvain (UCL). We thank Patrick Bogaert, Eric Le Boulengé, Xavier Draye, Fréderic Farnir and the referees for their comments on the manuscript.

\section{REFERENCES}

[1] Ardlie K., Liu-Cordero N.S., Eberle M.A., Daly M., Barrett J., Winchestcer E., Lander E.S., Kruglyack L., Lower-than-expected linkage disequilibrium between tightly linked markers in humans suggests a role for gene conversion, Am. J. Hum. Genet. 69 (2001) 582-589.

[2] Baret P.V., Hill W.G., Gametic disequilibrium mapping: potential applications in livestock, Anim. Breed. Abstr. 65 (1997) 309-319.

[3] Boichard D., Maignel L., Verrier E., Analyse généalogique des races bovines laitières françaises, INRA Prod. Anim. 9 (1996) 323-335.

[4] Cardon L.R., Bell J.I., Association study desigs for complex diseases, Nat. Rev. Genet. 2 (2001) 91-99.

[5] Christakos G., Random field models in earth sciences, Academic Press, San Diego, CA, USA, 1992.

[6] Devlin B., Risch N., A comparison of linkage disequilibrium measures for finescale mapping, Genomics 29 (1995) 311-322.

[7] Farnir F., Coppieters W., Arranz J.J., Berzi P., Cambisano N., Grisart B., Karim L., Marcq F., Moreau L., Mni M., Nezer C., Simon P., Vanmanshoven P., Wagenaar D., Georges M., Extensive genome-wide linkage disequilibrium in cattle, Genome Res. 10 (2000) 220-227.

[8] Haley C.S., Advances in QTL mapping, in: Dekkers et al. (Eds.), Proceedings of the conference "From Lush to Genomics. Visions for animal breeding and genetics", Iowa State University, 1999.

[9] Hedrick P.W., Gametic disequilibrium measures: proceed with caution, Genetics 117 (1987) 331-341.

[10] Hill W.G., Robertson A., Linkage disequilibrium in finite populations, Theor. Appl. Genet. 38 (1968) 226-231.

[11] Jorde L.B., Watkins W.S., Carlson M., Groden J., Albertsen A., Thliveris A., Leppert M., Linkage disequilibrium predicts physical distance in the Adenomatous polyposis coli region, Am. J. Hum. Genet. 54 (1994) 884-898.

[12] Lewontin R.C., The interaction between selection and linkage. I. General considerations; heterotic models, Genetics 49 (1964) 49-67.

[13] Lewontin R.C., On the measures of gametic disequilibrium, Genetics 120 (1988) 849-852.

[14] Lynch M., Walsh B., Genetics and analysis of quantitative traits, Sinauer Associates, Inc., Sunderland, MA, USA, 1998. 
[15] McRae A.F., McEwan J.C., Dodds K.G., Wilson T., Crawford A.M., Slate J., Linkage disequilibrium in domestic sheep, Genetics 160 (2002) 1113-1122.

[16] Moureaux S., Boichard D., Verrier E., Utilisation de l'information généalogique pour l'estimation de la variabilité génétique de huit races bovines laitières françaises d'extension nationale ou régionale, $7^{\mathrm{e}}$ Rencontre Recherche Ruminants, Paris, France (2000).

[17] Nagamine Y., Haley C.S., Sewalem A., Visscher P.M., Quantitative trait loci variation for growth and obesity between and within lines of pigs (Sus scrofa), Genetics 164 (2003) 629-635.

[18] Reich D.E., Cargili M., Molk S., Ireland J., Sabti P.C., Richter D.J., Lavery T., Douyoumjian R., Farhadian S.F., Ward R., Lander E.S., Linkage disequilibrium in the human genome, Nature 411 (2001) 199-204.

[19] Riley A.M., Hallas M.E., Lewontin R.C., Distinguishing the forces controlling genetic variation at the Xdh locus in Drosophila pseudoobscura, Genetics 123 (1989) 359-369.

[20] Sved J.A., Linkage disequilibrium and homozygosity of chromosome segments in finite populations, Theor. Pop. Biol. 2 (1971) 125-141.

[21] Tenesa A., Knott S.A., Ward D., Smith D., Williams J.L., Visscher P.M., Estimation of linkage disequilibrium in a sample of the United Kingdom dairy cattle population using unphased genotypes, J. Anim. Sci. 81 (2003) 617-623.

[22] Vignal A., Milan D., SanCristobal M., Eggen A., A review on SNP and other types of molecular markers and their use in animal genetics, Genet. Sel. Evol. 34 (2002) 275-305.

[23] Zapata C., Visedo G., Gametic disequilibrium and physical distance, Am. J. Hum. Genet. 57 (1995) 190-191.

[24] Zapata C., Carollo C., Rodriguez S., Sampling variance and distribution of the $D^{\prime}$ measure of overall gametic disequilibrium between multiallelic loci, Ann. Hum. Genet. 65 (2001) 395-406.

[25] Zapata C., Rodriguez S., Visedo G., Sacristan F., Spectrum of non-random associations between microsatellite loci on human chromosome $11 \mathrm{p} 15$, Genetics 158 (2001) 1235-1251.

[26] Zapata C., Nurez C., Velasco T., Distribution of non-random associations between pairs of protein loci along the third chromosome of Drosophila melanogaster, Genetics 161 (2002) 1539-1550. 